\title{
"MINHAS IMPRESSÕES SOBRE ZOLA" DE GEORGE MOORE
}

Thaís Marques Soranzo (tradutora) ${ }^{1}$

Resumo: Na busca de seu próprio caminho como escritor, o autor irlandês George Moore teve Zola como uma das principais referências na sua formação. A relação conturbada com o escritor francês foi retratada em "My Impressions of Zola" (1894), texto cuja tradução ao português brasileiro é pela primeira vez aqui apresentada.

Palavras-chave: George Moore, Émile Zola, My Impressions of Zola, Naturalismo.

1 Mestranda em Teoria e História Literária pela Unicamp, com apoio da FAPESP.

E-mail: thais.soranzo@gmail.com. 


\section{“MY IMPRESSIONS OF ZOLA”, BY GEORGE MOORE}

ABSTRACT: In searching for his own path as a writer, the Irish author George Moore found Zola to be one of the most important references in his career. The complex relationship with the French writer was described in "My Impressions of Zola" (1894), the Brazilian Portuguese translation of which is presented here for the first time.

Keywords: George Moore, Émile Zola, My Impressions of Zola, Naturalism.

\section{APRESENTAÇÃO}

"Não fui a Oxford ou a Cambridge, mas frequentei o Nouvelle Athènes" ${ }^{2}$ " (MOORE, 2007: 156); essa era uma declaração conhecida de George Moore (18521933), autor irlandês que, apesar de ter vivido em Londres, foi frequentador assíduo dos círculos culturais de Paris, onde conviveu com as principais personalidades da época. Aos vinte e um anos, muda-se para a capital francesa a fim de buscar sua própria identidade, ou, como ele próprio definiu, "para criar o meu eu"3 (2007: 5). Nos dez anos que permaneceu em Paris, Moore arrisca-se como pintor e, em seguida, como poeta, mas não tem grande êxito em nenhuma das atividades, descobrindo sua verdadeira vocação como romancista.

Todavia, o percurso pelo gênero romanesco não deixou de ser conturbado. Na busca de um estilo próprio de escrita, o movimento naturalista promovido por Émile Zola a partir da segunda metade do século XIX parece ter despertado em Moore a possibilidade de um caminho a ser seguido. A proposta de uma nova arte fundada nos métodos científicos, a qual buscasse sobretudo a "verdade" e o "conhecimento absoluto" (ZOLA, 1979: 35), deslumbrou o autor ainda iniciante, conforme ele atesta na autobiografia Confessions of a Young Man (1888):

A ideia de uma nova arte baseada na ciência, em oposição à arte do mundo antigo que se baseava na imaginação, uma arte que deveria explicar todas as coisas e abarcar a vida moderna na sua totalidade, nas suas ramificações intermináveis, ser, por assim dizer, uma nova crença em uma nova civilização,

2 "I did not go to either Oxford or Cambridge, but I went to the Nouvelle Athènes". Salvo quando indicado nas referências bibliográficas, os trechos aqui reproduzidos são de tradução minha.

3 "to create myself". 
me encheu de entusiasmo, e fiquei sem reação frente à grandeza da concepção e ao tamanho da ambição ${ }^{4}$. (2007: 49)

Fascinado pela estética naturalista, Moore logo tomou Zola como ídolo. Em abril de 1878, avistou o escritor francês pela primeira vez ao acompanhar Édouard Manet a um baile à fantasia em comemoração à adaptação teatral do romance $A$ Taberna [L'assommoir], então em cartaz no teatro parisiense Ambigu. No entanto, a amizade entre os dois se deu somente alguns meses depois, quando Moore finalmente tomou coragem de visitar Zola em sua casa de campo em Médan. A relação com Zola foi retratada por Moore no texto aqui traduzido "My Impressions of Zola", publicado em janeiro de 1894 na The English Illustrated Magazine e posteriormente incluído na segunda edição da coletânea de ensaios, Impressions and Opinions (1913).

No texto, temos o relato de dois encontros que aconteceram em Médan: o primeiro ocorreu alguns meses depois do baile, quando Moore estava ansioso para conhecer Zola e ao mesmo tempo intimidado pela presença do mestre, preocupado em lhe causar uma boa impressão; o segundo, em 1888, em um momento de grave desavença entre os dois.

O desentendimento teve como causa as opiniões expressas por Moore na autobiografia aqui já citada, Confessions of a Young Man, escrita alguns anos após seu retorno a Londres. Nela, Moore relata a experiência de Paris, com comentários acerca das personalidades com quem conviveu e dos principais movimentos artísticos e literários que lá vigoravam. Conforme observamos, ele registra o seu encantamento quando se deparou pela primeira vez com os pressupostos naturalistas. Não obstante, a empolgação com a literatura de Zola não tarda a esmorecer. Da mesma maneira que enfatizou o primeiro arrebatamento com as ideais do autor francês, também não poupou críticas à literatura de Zola quando passou a olhá-la de forma bem mais severa:

O que reprovo em Zola é sua falta de estilo [...]. Ele busca a imortalidade na descrição exata da loja de um comerciante de tecidos; se a imortalidade for concedida ao estabelecimento, ela deve ser atribuída ao comerciante, que o fundou, e não ao romancista, que o descreveu ${ }^{5}$. (2007:64)

\footnotetext{
4 "The idea of a new art based upon science, in opposition to the art of the old world that was based on imagination, an art that should explain all things and embrace modern life in its entirety, in its endless ramifications, be, as it were, a new creed in a new civilization, filled me with wonder, and I stood dumb before the vastness of the conception, and the towering of the ambition".

5 "What I reproach Zola with is that he has no style [...]. He seeks immortality in an exact description of a linendraper's shop; if the shop conferred immortality it should be upon the linendraper who created the shop, and not on the novelist who described it."
} 
Antes de serem publicados em livro, os capítulos de Confessions estavam sendo serializados na La Revue Independante. Moore, imaginando a revolta de Zola, tentou preveni-lo quanto ao teor de suas críticas, mas a discussão foi inevitável. Em um primeiro momento, ele tenta evitar a conversa, arrependido de ter ido até Zola para se justificar; no entanto, quando o autor francês terminou de ler em voz alta as críticas contidas em Confessions, Moore desabafou:

Você não percebe, meu caro amigo, que esse livro não é a minha verdadeira opinião sobre a vida e sobre as coisas, mas sim uma tentativa de colocar em palavras as fantasias fugazes da minha mente, o seu funcionamento íntimo, as suas mudanças de cor? Você nunca considerou mudar de opinião sobre as coisas? Encontrar a sua mente em meio ao caos da contradição?

A resposta de Zola foi categórica: "Não, não mudo de opinião facilmente. [...] Para mim, uma opinião é como um móvel pesado; é movido com dificuldade”. Era difícil para Zola compreender aquele artista ainda em formação. As impressões de juventude retratadas em Confessions mostram George Moore buscando o seu próprio caminho em meio a várias tendências, como se pode constatar no início do livro: "Aparentemente vim ao mundo com uma natureza semelhante a uma fina camada de cera, sem impressões, mas capaz de carregar todas elas; de ser moldada em qualquer formato"6 (2007: 01).

O naturalismo foi de fato uma fase importante do seu amadurecimento como escritor, mas logo Moore passou a ser mais cético quanto a essa estética. Muitos críticos já apresentam certa cautela em fazer uma associação imediata entre Moore e o naturalismo, mais especificamente entre o autor irlandês e Zola. Lilian Furst (1974), por exemplo, defende que os trechos de Confessions que retratam o entusiasmo pela estética naturalista devem ser lidos pelo viés da ironia (p.142). Ademais, para enfatizar o distanciamento entre Moore e Zola, a autora relembra passagens de "My Impressions of Zola" em que o escritor francês é alvo de deboche (pp.144145).

De fato, é impossível negar o caráter irônico de Confessions, especialmente por ser uma autobiografia em que Moore, já quase aos quarenta anos, retrata seus impulsos juvenis. Ao lembrar de sua empolgação com o naturalismo, o autor não pôde deixar de fazer uma ressalva bastante maliciosa: "Se alguém tiver curiosidade em buscar nos próprios livros a razão de tamanho êxtase, não encontrará nada - é como tomar o resto do champagne de ontem"7 (2007:50).

6 "I came into the world apparently with a nature like a smooth sheet of wax, bearing no impress, but capable of receiving any; of being moulded into all shapes".

7 If any one should be tempted to turn to the books themselves to seek an explanation of this wild ecstasy, he would find nothing - as well drink the dregs of yesterday's champagne. 
Em "My Impressions of Zola", também abundam críticas ao escritor francês e à sua literatura. Além de reprochar o mau gosto de Zola e seus gastos excessivos com uma "decoração vulgar”, Moore condena seu caráter mercenário e sua necessidade de fama. Para o autor, a preocupação de Zola em enriquecer faz com que ele escreva em um ritmo frenético, sem necessariamente refletir sobre a composição dos seus romances. Por conta disso, Moore acredita que os romances zolianos, para agradar ao homem mediano, apresentam uma "semelhança impressionante com os jornais populares”.

Levando-se esses comentários em consideração, seria possível admitir que Moore rompeu de vez com Zola e, por conseguinte, com a estética naturalista. Não obstante, não é por acaso que o texto de 1894 intitula-se "Minhas Impressões sobre Zola”. Ao afirmar que o texto apresenta um "tom mordaz" em relação ao autor francês (1974: 144), Furst parece menosprezar as outras impressões registradas por Moore, a começar pela insegurança em falar pela primeira vez com o mestre. Em seguida, após ouvir o que Zola tinha a dizer sobre as críticas contidas em Confessions, Moore confidencia: "Eu tinha lágrimas nos olhos. Nunca senti de forma tão aguçada a minha inferioridade; aquele homem era gigante na sua simplicidade”. Os encontros com Zola foram tão marcantes, que Moore faz questão de assinalar o quanto essa lembrança ainda o abala:

Narrei esses dois encontros com certa leviandade, mas na época eu estava profundamente abalado, e estou ainda hoje, pois será que é sempre certo expor os seus sentimentos e publicar suas opiniões conforme elas surgem na cabeça? $\mathrm{Ou}$ será melhor considerar uma opinião como um móvel pesado que é movido com dificuldade.

Ademais, além de admitir que a imaginação de Zola é "poderosa" e "fecunda", e que é “uma das mais extraordinárias que já ganhou expressão na literatura”, Moore encerra o texto com um tom melancólico, mas que não deixa de conter um certo respeito ao seu antigo mestre. Ao imaginar que fim levaria Zola, ele se questiona se não iria se arrepender de ter se afastado do autor: “[...] vamos nos lamentar sobre a sua vida e o seu trabalho, e sobre o erro que cometemos".

Assim, o texto aqui apresentado nos revela duas personalidades fundamentais para a literatura. Ele não deixa de ser um tributo a alguém que foi tão importante na trajetória de Moore. Suas impressões podem mudar, mas como é possível retratar uma figura como Zola de forma unívoca? Essas alternações estão presentes na própria escrita de Moore. Em mais de uma ocasião, ele interrompe raciocínios ou acrescenta divagações, o que tentamos, na medida do possível, preservar na tradução. 


\section{TRADUÇÃO}

Manet me convenceu a ir ao bal de l'Assommoir ${ }^{8}$ vestido como um operário parisiense $^{9}$, pois ele gostava de incongruências, e a camisa e o boné, combinados à minha aparência e ao meu sotaque, atiçaram a sua imaginação. "Não há nenhum francês em Londres que ocupe a mesma posição que você em Paris", ele disse, e eu refleti sobre essas palavras enquanto o acompanhava pela tout Paris ${ }^{10}$ reunida na Elysée Montmartre para o baile à fantasia em homenagem à peça que estava sendo encenada no Ambigu. "Mas preciso lhe apresentar a Zola. Aí está ele", exclamou ao apontar para um homem grande e corpulento em traje social, já que, como afirmou Manet, não se pode esperar que um escritor sério se vista a caráter.

Zola fez uma reverência e seguiu adiante, nos desprezando um pouco; Manet teria gostado de ver seu embaraço ao conhecer alguém, e continuamos andando, cientes do nosso fracasso, eu pensando em quão prazeroso teria sido ir com esses dois a um canto e falar sobre arte por meia hora, "e que lembrança maravilhosa teria sido!”, pensei, e logo implorei a Manet para procurar Zola comigo. Mas ele não era encontrado em lugar algum.

"Ele deve ter voltado para casa", eu disse, e Manet respondeu: "Não importa. Você vai achá-lo quando quiser na casa em Médan”.

Por uma razão ou outra, só alguns meses depois criei coragem e peguei o trem na Saint-Lazare. Não há nenhuma estação em Médan, a mais próxima é a - (consultem os curiosos a tabela horária, pois esqueci o nome de lá) - e Médan é um povoado habitado apenas por camponeses, cerca de dois quilômetros da estação. Algo levou Zola àquele lugar e, por precisar de uma casa de campo, comprou a de um camponês, a qual ele acabou de transformar em uma espécie de castelo; me pareceu um lugar feio, uma grande parede de tijolos vermelhos com uma porta pequena, através da qual me levaram ao interior da casa e me deixaram esperando em uma sala de bilhar.

Não foi Zola quem me recebeu, mas Madame Zola. Ela havia se esquecido de mim, embora tivéssemos nos conhecido no estúdio do Manet, e foi só depois de várias explicações tediosas que ela, ainda um tanto relutante, me guiou pela casa até uma escadaria polida de carvalho, estreita e íngreme. Na parede do último lance de escadas, havia gravuras japonesas retratando fornicações selvagens; um anúncio bastante óbvio, pensei, do naturalismo - mas elas foram logo esquecidas, pois dentro de alguns segundos eu estaria na presença do mestre. Ela abriu a porta e foi embora, e eu me encontrei em um lugar tão grande quanto o estúdio de um Acadêmico, iluminado por uma claraboia e por uma janela enorme. Cheguei por um instante a me perder no meio dos móveis maciços, e foi somente após eu passar por um atril que encontrei o mestre revisando provas em um sofá próximo à janela.

8 "baile d'A Taberna".

9 Moore vestiu-se como Coupeau, personagem de L'Assommoir.

10 "alta sociedade francesa". 
Ele não se levantou para me cumprimentar, mas se contentou em descruzar a perna gorda e indicar com a mão uma cadeira. Seu modo era terrivelmente distante e frio, e o meu constrangimento aumentou, porque de súbito lembrei que tinha ouvido dizer que Zola não demorava a se decidir se estava falando com um idiota ou com um homem de espírito, e que em menos de um minuto o idiota era mandado embora de forma brusca. "E ele me tomou por um idiota, embora eu não tenha dito uma palavra”. Corri os olhos pelo terrível mestre estendido no sofá, os óculos no nariz, lendo-me, adivinhando os clichês que eu estava tentando evocar. "Se Homero e Shakespeare fossem de repente apresentados, eles teriam que começar a conversa com comentários sobre o clima ou sobre o prazer que cada um encontrou no trabalho do outro", eu disse a mim mesmo, "e se esse homem ao menos me desse a liberdade que ele daria a Shakespeare ou a Homero, eu poderia pensar em algo mais interessante do que os elogios que estou soltando sem parar".

Zola não era naquela época o que ele é agora, um homem bondoso e gentil, com o costume de receber todos que queiram visitá-lo e de responder a qualquer pergunta. Ele era então o iconoclasta, o destruidor de ícones, um urso que amaldiçoava o universo e mandava todos os visitantes embora. Ainda assim, seus escritos exalavam uma certa generosidade e simpatia, e eu sempre senti, enquanto lia seus artigos semanais no The Voltaire, que nós nos entenderíamos. Imaginei que quando fosse visitá-lo, ele viria até mim, os braços estendidos em um gesto benevolente, e me tomaria no mesmo instante como seu confidente. Esse Buda deitado no sofá, ajeitando de tempos em tempos os óculos naquele nariz pequeno e estranhamente quadrado, estava em tal desacordo com o meu sonho que eu mal podia acreditar, durante aquele momento terrível em que eu tentava improvisar elogios, que este era o meu Zola. Nenhum dos comentários produziu o menor efeito, não mais que a água escorrendo sobre um bloco de granito, e fervorosamente busquei um assunto para engatar a conversa, alguma coisa, não importa o quê, que pudesse interessá-lo.

Naquela época, o poder exercido pelas bibliotecas itinerantes sobre a literatura me preocupava muito, então fui direto ao assunto, agarrando-me à primeira frase de transição.

"A posição do romancista na Inglaterra é a de um escravo", eu disse, "pois lá os livros não são comprados, mas sim alugados ${ }^{11}$ ”.

"Mas se um homem escreve um livro que interessa ao público, o público vai encontrá-lo".

"O público irá encontrá-lo na hora certa, sem dúvida”, respondi, "mas enquanto isso o homem morre de fome”.

${ }^{11}$ Moore refere-se aqui às bibliotecas itinerantes Mudie's Select Library e W.H Smith, cujos donos, Charles Mudie e W.H. Smith, detinham o monopólio do comércio de livros. Além de recusar os títulos que considerassem imorais, os livreiros adotavam o formato do romance em três volumes. Como tinham preços excessivamente caros, um leitor de classe média não teria condições de comprá-los, restando-lhe a alternativa de emprestar, mediante o pagamento de uma taxa, apenas um livro por vez (SHOWALTER, 1993: 31). 
"Sim, é provável; e seu problema não é pouca coisa, um comerciante sempre entre você e seu público".

"Ah, ele está começando a perceber que afinal eu não sou um idiota”, disse a mim mesmo, e assim que expliquei o poder atribuído a Mudie e a Smith por conta do seu monopólio, habilmente mudei a conversa da questão prática para a questão moral, fazendo alguns comentários desdenhosos sobre o Puritanismo como influência artística. Zola, que já vinha despertando da sua letargia, estava agora totalmente acordado.

"O que você está dizendo", declarou o grande homem, "é extremamente interessante. Escrevi um artigo sobre a influência do Protestantismo na arte; sairá amanhã no Figaro, e nele eu afirmo que o Protestantismo nunca produziu arte de qualidade. Milton é o único escritor protestante. Os elisabetanos Shakespeare e Jonson viveram antes de o Protestantismo controlar o espírito nacional, o gênio da nação e assim por diante".

A conversa então se tornou amigável e prazerosa. Zola me perguntou sobre George Eliot; qual dos seus romances eu considerava ser o melhor? Com qual escritor francês ela se parecia? Embora eu sentisse que estava arriscando a reputação que acabara de ganhar, tive que admitir que não conseguia pensar em alguém que pudesse ser comparado a ela. A conversa teve uma pausa e, para minha surpresa e satisfação, Zola começou a me contar sobre o novo romance que estava escrevendo. Devemos ter conversado por cerca de três quartos de hora, e então, com receio de prolongar demasiado minha visita, disse adeus ao mestre. Ele me acompanhou até lá embaixo, entusiasmado durante todo o tempo, e pediu para que eu tornasse a visitá-lo. Ali eu sabia que tinha feito um amigo. "Fiz um amigo", repeti a mim mesmo enquanto a carruagem movia-se pelo campo francês, verde e plano, meus olhos observando o mistério daquele horizonte baixo e distante, os álamos apontando para as primeiras estrelas. Um trem apitou em meio a solidão. "Fiz um amigo", repeti a mim mesmo ao ouvir o tinido ao longe, e quando o barulho transformou-se em ecos suaves, absorvidos um por um pela noite escura da longa e baixa planície, eu disse: "Sim, ele é exatamente o homem que imaginei quando li seus artigos. Uma mente clara e equilibrada, uma natureza benevolente, intenso quanto às suas convicções, fiel a suas opiniões. Um pouco rude no começo; talvez o que eu tenha tomado por grosseria fosse apenas timidez; ademais, não deve ser divertido ouvir alguém dizer diretamente que você é um grande autor. Eu mesmo não iria gostar".

Anos se passaram. Escrevi vários livros. A Mummer's Wife ${ }^{12}$ foi traduzido para o francês; publicado no The Voltaire e no Vie Populaire. Charpentier iria editá-lo

${ }^{12}$ Considerado pela crítica como a tentativa de Moore em inserir o naturalismo francês na ficção inglesa (FRAZIER, 2011: 5), A Mummer's Wife (1885) fora banido pelos livreiros. À época da publicação do romance, Moore mantinha uma correspondência constante com Zola e engajava-se, junto ao editor Henry Vizetelly, em publicar os romances do autor francês na Inglaterra. Como contribuição para a divulgação de Zola, Moore escreveu prefácios elogiosos às traduções inglesas dos romances Roupa Suja [Pot-Bouille] e O Regabofe [La Curée], publicados pela editora de Vizetelly em fevereiro e novembro de 1885 , respectivamente. 
em forma de livro e Zola prometeu escrever o prefácio. Confessions of a Young Man estava sendo publicada na La Revue Independante, e a notícia que chegou ao estrangeiro foi a de que o próximo episódio conteria um ataque mordaz ao A Terra [La Terre]. Escrevi a Zola alegando que aquilo não era verdade e propondo passar a segunda-feira com ele. "Na segunda-feira de manhã você vai receber o novo número da Revue, e aí poderemos discutir o assunto no café da manhã”. Eu sabia que o número continha - bem, algumas afirmações irrelevantes sobre o naturalismo; eu tinha esperança de conseguir explicá-las. Mas não me sentia de todo confiante, então pedi ajuda ao fiel Alexis - o temperamento tranquilo do corpulento Alexis serviria como um apaziguador quando a discussão apertasse. Mesmo que tudo acabasse bem, era improvável que não houvesse momentos em que - acho que não terminei a frase na época; não vou me preocupar em fazer isso agora.

Caminhamos em volta do rio, que resplandecia como uma seda ondulada em meio às margens verdes, repletas da exuberância de junho, e mais adiante o verde campo francês parecia se regozijar com a luz do sol como uma criatura viva. Passeamos enquanto falávamos dos nossos livros, e eu senti um prazer especial por aqueles álamos altos e eretos que se distinguiam na planície, e pelas nuvens brancas suspensas entre as árvores. E quando a lembrança de Zola interrompeu meus devaneios, contei ao Alexis exatamente o que eu havia escrito, e o caro colega me assegurou que Zola não poderia ficar ofendido com uma crítica tão banal.

"Sim, Alexis, mas você sempre diz o que é agradável ouvir".

Como da outra vez, Zola estava estendido no sofá próximo à janela e, após algumas palavras de cumprimento, disse:

"Receio, meu caro amigo, que não poderei escrever o prefácio. Você fez com que isso se tornasse impossível para mim".

Quando analisadas de perto, as frases que eu utilizara se mostraram bem mais difíceis de explicar do que imaginei. A discussão foi penosa, e a campainha para o café da manhã foi um alívio bem-vindo. "Pelo menos acabou", disse a mim mesmo; mas para o meu terror, em vez de corresponder ao meu pensamento, o mestre antecipou:

"Vamos descer para o café da manhã, mas depois retomaremos o assunto detalhadamente; lerei as passagens em voz alta para você".

"Céus!", pensei, "era melhor que eu não tivesse vindo".

Depois do café da manhã, Zola, Alexis e eu caminhamos pelo jardim e falamos sobre coisas insignificantes por cerca de uma hora ou mais. Em seguida, Zola disse:

"Agora vamos subir". Ele foi na frente e eu o segui, com um sentimento muito parecido ao da época da escola, quando era castigado com chicotadas. O mestre deitou-se no sofá; eu me sentei numa cadeira pequena; ele afirmou: "Você ficará mais confortável em uma cadeira maior".

As passagens já tinham sido marcadas e me foram lidas por uma voz baixa e deliberada. Eu escutei, pensando em qual seria a melhor saída para me defender; Zola comentou cada um dos sarcasmos. 
"Como posso escrever seu prefácio depois disso? Eu bem que gostaria, você sabe, mas lhe pergunto como poderia? Ouça!"

"Você não percebe, meu caro amigo, que esse livro não é a minha verdadeira opinião sobre a vida e sobre as coisas, mas sim uma tentativa de colocar em palavras as fantasias fugazes da minha mente, o seu funcionamento íntimo, as suas mudanças de cor? Você nunca considerou mudar de opinião sobre as coisas? Encontrar a sua mente em meio ao caos da contradição?”

"Não", respondeu, "não mudo minha opinião facilmente. Veja o Alexis" (de fato ele estava ali, redondo como um barril e como sempre com um cigarro entre os dentes); "faz vinte e cinco anos que conheço o Alexis, e o que acho dele agora é exatamente igual ao que sempre achei. Para mim, uma opinião é como um móvel pesado; é movido com dificuldade".

"Mas", argumentei, "as passagens que você acabou de ler são de um capítulo intitulado 'La Synthèse de la Nouvelle Athenes'...e devem ser consideradas como a expressão das opiniões dos vários ratés ${ }^{13}$ que lá se reúnem”.

"Vou considerar isso como uma legítima defesa, mas repare que as opiniões aludidas no café coincidem exatamente com aquelas que você defende na primeira parte do livro".

Tive que recorrer à defesa anterior, a de que um homem muda, logo seus pensamentos contraditórios não podem ser tomados como as opiniões que ele defende e nas quais se mantém.

"Com que frequência ouvimos cristãos fazerem piada com o Cristianismo?", considerei o argumento barato, mas Zola não reparou. Ele seguiu com a leitura:

"Depois do que você escreveu sobre o Goncourt", afirmou, "você nunca mais poderá visitá-lo ${ }^{14 "}$.

"E nem quero; ele não é meu amigo".

"Os discípulos, a vaidade infantil, o bric-à-brac ${ }^{15}$, até mesmo a acusação de se autopromover nos jornais com a morte do irmão, tout est là, rien ne manque ${ }^{16}$. $\mathrm{O}$ que você diz sobre mim não é nada comparado ao que você diz sobre o Goncourt”. Apressei-me em concordar com essa opinião, mas Zola não queria ser bajulado. "Não, meu caro amigo", declarou de forma grave e triste, "você não chama o seu

13 "fracassados"; o café Nouvelle Athènes, localizado na Place Pigalle, em Paris, era muito frequentado por artistas da época. No capítulo VIII de Confessions, Moore menciona, dentre outros, Villiers de l'Isle-Adam, Manet, Degas e Stendhal.

$14 \mathrm{Na}$ passagem aludida por Zola, Moore faz uma crítica severa a Edmond de Goncourt, alegando que ele não é um artista, pois está unicamente preocupado em tornar-se imortal. Segundo Moore, para atingir tal objetivo, os irmãos Goncourt escrevem sobre tudo, sem deixar nada escapar, pois é possível que "apenas uma palavrinha possa lhes conferir a imortalidade" (MOORE, 2007: 67). 15 "bricabraque": conjunto de objetos antigos, usualmente acumulados por colecionadores. Dentre os motivos de deboche de Moore, está o fato de os irmãos terem desejado que esses objetos fossem vendidos após sua morte para arrecadar fundos na criação de um prêmio literário, o que, na visão do autor irlandês, reforçava a ideia de que os Goncourt criavam diferentes estratégias apenas para ganhar fama (Ibidem).

16 "está tudo aqui, não falta nada". 
livro de Memoirs d'un Jeune Anglais ${ }^{17}$, você diz Confessions d'un Jeune Anglais ${ }^{18}$, e quando usamos a palavra Confissão, queremos dizer que finalmente falaremos a verdade. Retomei essas páginas destacando as expressões utilizadas, não porque estou bravo, mas porque quero convencê-lo de que você tornou impossível que eu escreva o prefácio de A Mummer's Wife. O que você pensa de mim não me afeta, não, não estou falando isso; somos velhos amigos. O que você afirma sobre mim me afeta, quero dizer, nada do que você afirme possa abalar minha posição...Você admite no livro que me deve a sua primeira inspiração. Tenho orgulho disso e lhe agradeço por expressá-lo. Sinto muito se mudou de opinião; afinal, é aquela eterna lei os filhos devoram os pais. Não reclamo. A natureza quis assim”.

Pronunciou essas palavras com tristeza enquanto andava pelo aposento. $\mathrm{O}$ cair da tarde se aproximava, a vasta mobília emergia feito sombras. Eu tinha lágrimas nos olhos. Nunca senti de forma tão aguçada a minha inferioridade; aquele homem era gigante na sua simplicidade. "Ele é melhor que seus livros", pensei comigo mesmo, "e isso é uma grande coisa, já que escreveu livros muito bons".

Narrei esses dois encontros com certa leviandade, mas na época eu estava profundamente abalado, e estou ainda hoje, pois será que é sempre certo expor os seus sentimentos e publicar suas opiniões conforme elas surgem na cabeça? Ou será melhor considerar uma opinião como um móvel pesado que é movido com dificuldade. Alexis havia se dedicado durante meses na revisão da tradução que seria publicada por Charpentier e aguardava o prefácio de Zola como recompensa do seu esforço, mas bastaram algumas palavras minhas para arruinar suas esperanças. Ele não me repreendeu por ter dado prejuízos financeiros; apenas alegou, "C'est Charpentier que va boire um bouillon. Mille francs de corrections" 19 . O campo verde-cinzento estendia-se diante de nós, plaino e obscuro - uma massa escura de árvores à nossa frente, um álamo destacando-se em meio à vasta planície. Alexis dissertava enquanto caminhávamos pelo campo solitário, mas não o escutei. O tempo todo eu pensava nas últimas palavras de Zola ao se despedir: "Espero que entenda que as nossas relações pessoais continuam as mesmas, apenas que você tornou impossível que eu escreva o prefácio”.

Naquela época Zola era um homem gordo; pouco tempo depois emagreceria. Ao cortar a bebida durante as refeições, perdeu dezesseis quilos em dois meses. Parece que ele aceitou a máxima de Balzac de que a elegância da vida está principalmente na cintura. Conforme sua cintura afinou, seu modo de vida tornou-se mais expansivo. Já não é mais o homem recluso de Médan; construiu uma torre na sua casa de campo - com qual intenção eu nunca entendi - e mora numa mansão espaçosa na Rue de Bruxelles, a qual mobiliou com gravuras de carvalho, tapeçarias, retratos de arcebispos e corrimão de ferro forjado. Há uma estátua de gesso da Vênus de Milo na balaustrada da escada. A casa parece revelar uma mente vulgar, uma espécie de

${ }_{17}$ Memórias de um jovem inglês

${ }^{18}$ Confissões de um jovem inglês

19 "Será o Charpentier quem terá prejuízos. Mil francos pelas revisões". 
rede tecida de forma grosseira por cujos fios escapam todas as coisas vivas, e a qual traz à superfície

apenas um acúmulo de débris ${ }^{20}$. "Da Rue de Lafayette", eu disse. Por que ele acha importante colecionar todas essas coisas? Grandes artistas não precisam de bric-à-brac. Manet viveu toda a sua vida em meio a um mobiliário vermelho e luxuoso; e não tenho certeza se deveria ter comentado sobre a mobília de Zola (ela já não foi descrita por jornalistas e reproduzida em fotografias em todos os jornais ilustrados?), se não fosse pelo fato de que, ao modelar a cintura e acumular bric-àbrac bem genéricos, Zola passou por uma mudança mental decisiva. Uma vez ele disse que daria um baile. Acho que nunca executou seu plano. De toda forma, nos últimos três anos sua casa esteve aberta a vários visitantes, e ele respondeu a dez mil questões heterogêneas feitas por onze mil cento e cinquenta e sete entrevistadores com uma cortesia infalível e, devo admitir, com um bom senso excepcional.

Sua mente não é tão intensa ou penetrante quanto à de Turguêniev, mas é capaz de olhar para uma vasta superfície com uma lucidez admirável, e ele responde às perguntas mais tolas de forma ponderada. $\mathrm{O}$ carrinho de maçãs de uma senhora foi derrubado na Place de Clichy, e um repórter quis saber a opinião de Zola. Ele respondeu que não tem informações muito precisas quando o assunto refere-se a maçãs, mas acredita que seu cultivo é uma indústria importante no norte da França. Se os vendedores de maçãs de Montmartre são proibidos de expor seus produtos à venda, a liberdade individual é ameaçada, e uma indústria grande e importante corre o risco de ser afetada. Em contrapartida, as ruas não podem - e por aí vai. Mas até mesmo nessas banalidades ele acrescenta um toque de raro bom-senso. Esse toque eu não consigo reproduzir, é o segredo incomunicável da sua genialidade. Mas se algum leitor deste artigo tiver curiosidade em ouvir Zola, recomendo o livro Enquête Littéraire ${ }^{21}$, de Jules Huret. A entrevista de Huret com Zola é uma obra literária surpreendente. Nessa entrevista compreendemos, como é de se esperar depois de uma longa relação com o próprio Zola, que seu gênio não é nada mais do que o triunfo e a apoteose do bom-senso. Pois seu gênio não tem asas, não é capaz de alçar as estrelas; é mantido no que arrisco chamar de nível superior da mediocridade, e está sempre com ele, tanto nas pequenas quanto nas grandes ocasiões. Tome como exemplo a resposta a um repórter que o entrevistou no Savoy Hotel. Zola chegou tarde na noite anterior e havia acabado de se levantar. A pergunta era: "quais são suas impressões de Londres?" E a resposta: "Minha primeira impressão de Londres foi a de um apetite excelente. O trem estava atrasado, e só fomos jantar às nove horas, mas comemos extremamente bem”. É possível responder a uma questão tão tola de forma mais sensata?

Acabei de dizer que a visão de Zola sobre a vida não é tão intensa, penetrante ou sutil como a de Turguêniev. É totalmente diferente. A mente de Zola é patriarcal; ele é um herói do mundo antigo, um patriarca ultrapassado no século dezenove.

20 "entulhos; resíduos".

${ }^{21}$ Enquête sur l’ Évolution Littéraire [Investigação sobre a Evolução Literária], 1891. 
Nem mesmo Abraão, que vivia entre os seus rebanhos, manadas e vários escravos, via ou pensava de modo tão simples quanto ele. Há carruagens de aluguel, lavadeiras e châssepots ${ }^{22}$ na saga dos Rougon Macquart, mas são apenas atrações fortuitas que não afetam em nada o aspecto geral do trabalho. Hugo é considerado o último dos poetas do mundo antigo; mas a verdadeira diferença entre Zola e Hugo é que um é capaz, e o outro não, de escrever versos. Tire de Hugo o seu gênio para versificação e você terá o romancista. Ele teria escrito uma série de romances muito parecida à saga dos Rougon Macquart. Seriam vinte volumes, ou até mais, e teriam vendido tanto quanto. Privado da sua versificação, Hugo teria aceitado as carruagens de aluguel e as lavadeiras. Ele não poderia ter feito de outra maneira, pois os dois homens enxergavam a vida por fora e tendiam a exagerar o lado de fora. Não obstante, o hipotético trabalho teria sido diferente dos Rougon Macquart. Hugo era um artista mais natural do que Zola. A sua imaginação era rara, mas não tão poderosa e nem tão fecunda. A imaginação de Zola é uma das mais extraordinárias que já ganhou expressão na literatura. Pense, você que leu os vinte volumes, nas centenas de lugares que ele lhe mostrou e com os quais lhe familiarizou, da mesma forma que você está familiarizado com o aposento onde vive. Você não é capaz de ver a incestuosa Renée sonhando no seu boudoir amarelo, ou sendo arrebatada pelo calor da pele sob a sombra maligna das plantas tropicais na grande estufa? Aqueles mercados não ficam na sua memória - sempre o contorno dos telhados contra o céu pálido? O cheiro dos peixes e a centena de colorações do peixe; as crianças brincando com os arcos no meio dos pombos? $\mathrm{E}$ a cena em que as cabeças dos pombos são cortadas, em uma disputa para saber quantos desses animais cada sujeito consegue sangrar em uma hora? E como conhecemos intimamente o grande jardim de Paradou e a morte adorável de Albine, que morreu asfixiada pelas flores com as quais ela própria enfeitou o quarto; a enumeração das flores, a evocação de uma orquestra de aromas, já que cada fragrância lembra o som de um instrumento, e a última frase - "Albine morre no soluço supremo das flores" - que maravilha! ${ }^{23}$

E os estilos de vida, a árvore genealógica, as diversas maneiras pelas quais o animal humano ganha seu sustento! Quem não se lembra dos fabricantes de correntes de ouro em A Taberna e dos que bordavam casulas em $O$ Sonho [Le Rêve]? Em $O$ paraíso das damas [Au Bonheur des Dames], a atividade de todos os funcionários é explicada; o fenômeno de cada hora nos é revelado. Germinal é repleto de detalhes sobre a vida dos mineiros, as cordas, as roldanas, as fornalhas, os vagões, os cavalos. Mas é melhor eu conter a minha pena ou este artigo vai virar um mero catálogo.

Mas Zola forneceu essas evocações extraordinárias do exterior da vida humana com almas humanas? Ele criou personagens que não serão inferiores se comparadas às de Balzac? A evocação que Zola faz da alma é ínfima, quase sempre

22 Fuzil Chassepot, utilizado pelas tropas francesas no período de 1867 a 1874, especialmente durante a Guerra Franco-Prussiana. Disponível em: <https://bit.ly/2FvXhuE>. Acesso em 10 jan. 2019.

23 Moore refere-se aqui a cenas dos romances $O$ Regabofe, $O$ Ventre de Paris [Le Ventre de Paris] e O Crime do Padre Mouret [La Faute de L'Abbé Mouret], respectivamente. 
fragmentária e vaga. Uma alma aparece em Gervaise, e Coupeau também chega a ter uma, e pelas numerosas páginas vagamente surgem aqui e ali algumas sombras de almas humanas. Isso é tudo. Ao se tratar de almas, Gervaise representa seu melhor desempenho, e é por isso que considero A Taberna como seu melhor romance. Há outras razões. Quando escreveu A Taberna, Zola era mais do que nunca, e certamente mais do que seria depois, um discípulo de Flaubert. O romance é inteiramente escrito no estilo de Flaubert, as frases curtas incrementadas com epítetos imagéticos. Os antigos mestres acreditavam que a originalidade podia ser encontrada em sentimentos e percepções individuais, não no maneirismo, e como compartilho dessa opinião, acho lamentável que Zola não tenha continuado a escrever no mesmo estilo com o qual ele compôs seu melhor romance. Mas o estilo se tornou muito trabalhoso para ele, assim como o Pré-Rafaelismo se tornou muito trabalhoso para o Sr. John Millais, e, após A Taberna, seu estilo afrouxou, e a cada novo livro ele parece mais e mais inclinado a se entregar à comodidade da expressão redundante. Há passagens excelentes até mesmo nos seus piores romances, mas no que concerne ao meu gosto pessoal e ao meu interesse pelo seu trabalho, teria escolhido revisar edições dos seus trabalhos anteriores, em vez dos novos romances que ele está engajado em escrever - Lourdes, Paris, Rome etc.

Revisar as edições dos trabalhos de Zola! Como alguém pode facilmente falar besteiras. Seu método de escrever romances não admite revisões. É possível também perguntar ao editor de um jornal diário a respeito das expressões mais utilizadas nos artigos principais, nas notas sobre o teatro e nos boletins das corridas de barco. Nos últimos dez ou doze anos, criou-se uma semelhança impressionante entre os romances de Zola e os jornais populares. O romance e o jornal me parecem estar no mesmo patamar; a intenção dos dois é a mesma, e os meios empregados são os mesmos. É verdade que os boletins de Zola sobre a guerra Franco-Prussiana são melhores do que os boletins dos correspondentes de guerra do Daily Telegraph. Também é verdade que o cenário do "Lyceum" é mais bem elaborado do que o do "Surrey”, mas essa não é uma razão para confundir o cenário da peça Muito barulho por nada ${ }^{24}$ com as pinturas de Turner, Constable e Wilson, e encontramos essa mesma diferença entre os retratos da guerra em Guerra e Paz e em A Derrocada [La Débâcle] - não uma diferença de nível, mas sim de tipo. O romance de Zola é praticamente o jornal diário. Ele descobriu uma fórmula que agrada o homem mediano da mesma maneira que o Daily Telegraph ou o Petit Journal, e escolhe seus temas não a serviço de um instinto artístico, mas de acordo com o gosto do público. Trezentos mil peregrinos vão a Lourdes todo ano. Cada peregrino é por certo um leitor, e os aflitos em todos os países se interessam pela questão. Entre a crença e a descrença, ele vai encontrar um meio termo do mesmo modo que encontrou um meio termo entre a França e a Prússia. Ouvi ele se vangloriar, sem nem perceber o tamanho da importância artística do que estava dizendo, de que havia escrito um

24 Os teatros londrinos Lyceum e Surrey foram inaugurados, respectivamente, em 1765 e 1782 . A comédia shakespeariana aludida por Moore, Much Ado about Nothing, foi encenada no teatro Lyceum em 1882. 
romance francês sobre a guerra sem dar a Prússia qualquer motivo para ofensa. Considero que a imparcialidade sublime do verdadeiro artista é bem diferente da falsa imparcialidade do jornalista que quer gerar polêmica. O verdadeiro artista enxerga a vida como Deus, sem preconceitos; a vida é para ele - acho que a frase é de Flaubert - une hallucination à transporter ${ }^{25}$.

Zola me disse que tinha feito uns cálculos, e, considerando o feriado de quinze dias no Natal, Lourdes levaria sete meses para ser escrito. Quinhentas páginas em sete meses! Tolstói levou seis anos para escrever Anna Kariênina, dez para Guerra e Paz; Flaubert precisou de dezessete anos para escrever As Tentações de Santo Antão, oito ou nove para $A$ Educação Sentimental; e sete ou oito tradutores já estão trabalhando em Lourdes; irá aparecer no New York Herald, e o Sr. Bennet pagou mil libras pelos direitos da série. Mas as informações necessárias a respeito dos vários formatos e idiomas nos quais o romance aparecerá serão tema para um artigo sobre bibliografia. Basta aqui dizer que o romance renderá a Zola cerca de quatro mil libras antes de ser publicado por Charpentier em forma de livro; então será lido por todos, exceto pelos homens de letras - mas eles são tão poucos, que sua abstenção não afetará as vendas em termos materiais. Se o romance não vender trezentas mil cópias, será um fracasso, e se o romance seguinte sobre a Aliança Russa não vender quinhentas mil, será um fracasso. Algum grande escritor já olhou para a literatura sob esse ponto de vista?

A ideia da conquista parece inerente a Zola. Vinte e cinco anos atrás ele escreveu um romance intitulado A Conquista de Plassans [Le Conquête de Plassans]. A ideia surgiu de novo em A Obra [L'Oeuvre], e dessa vez foi Paris quem foi conquistada. E agora parece que Zola planeja a conquista do mundo. Ele veio à Inglaterra cercado por um exército de jornalistas; mísseis foram disparados no Crystal Palace, e trompetes foram tocados em sua homenagem na Mansion House. Provavelmente ele prossiga em uma missão parecida para São Petersburgo (já se falou sobre isso); talvez até visite a América. Por que não? Existem sessenta milhões de pessoas nos Estados Unidos que, por meio de traduções, poderão ler os Rougon Macquart! Os jornais relataram que Madame Zola, impressionada com a extensão dos nossos subúrbios londrinos, disse: "Esta é uma cidade que lhe seria conveniente, Emile". Cada casa representava para ela uma possibilidade de vender um romance, a edição de Charpentier, três francos e cinquenta. Se dissessem a Zola que um concierge não tivesse ouvido falar no seu nome, ele se sentiria desmotivado. Uma investigação teria início, e se uma associação de concierges fosse descoberta, ele trataria de marcar um encontro. Ele considera todos aqueles que não leem seus romances como perdedores. Estão perdendo o quê? Ah, isso não sei dizer; não a arte, pois a qualidade dos seus escritos parece que o preocupa tanto quanto a qualidade das coisas que ele compra. A madeira entalhada e as balaustradas de ferro podem não ser muito bem feitas, mas elas saem bem nas fotografias, e cada repórter é bem-vindo e cada viajante - chineses, peruanos, esquimós - todos são considerados como um público

25 "uma alucinação para ser carregada". 
em potencial, e as tardes são dedicadas a discutir qual a melhor maneira de colocar os livros à venda.

Algumas traduções do seu trabalho devem aparecer em todos os dialetos, e descobrir algum deles que ainda não tenha se restringido à escrita em papel, e conseguir que o primeiro trabalho impresso nesse dialeto seja uma tradução da série dos Rougon Macquart seria por certo um grande sucesso. O Sr. Bruneau ${ }^{26}$ chega com uma partitura que ele acabou de escrever para um dos romances, e o senhor do Paraguai dá um pulo e propõe que a ópera seja feita na língua de seu país; o tibetano talvez faça o mesmo. Bruneau e Zola articulam juntos. Viva! Outra forma de divulgação foi encontrada, e os termos do contrato foram discutidos. Só outro dia desses, em um artigo sobre dramas musicais, após equiparar Wagner e Bruneau, Zola explicou que ele criaria um drama musical com personagens humanos; e ao fazê-lo, "o colossal Wagner empalideceria no alto pedestal dos seus símbolos". Zola acredita que os jovens compositores franceses não escreveram música de qualidade porque seus libretos não eram humanos o suficiente. Em uma palavra, ele se imagina escrevendo vários libretos nos quais os jovens compositores franceses iriam acrescentar um pouco de música da mesma maneira que se acrescenta creme aos méringues $^{27}$. Bem, se alguém opina sobre todos os assuntos, vai chegar uma hora em que dirá asneiras. Receio que a hora de Zola tenha chegado!

O desejo pelo ouro para benefício próprio é compreensível em certa medida; mas Zola não tem amor pelo dinheiro, ele desperdiçou tudo o que ganhou em uma decoração vulgar e em uma arquitetura absurda. Os prazeres da vida o entediam de forma excessiva, ele diz então; mas receio que não tenha se familiarizado com eles. Dos prazeres da Arte é igualmente ignorante. A sua juventude, que isso seja dito em seu favor, foi difícil o suficiente para ter derrubado qualquer um, exceto os mais determinados. Todas as manhãs trabalhava em um romance durante quatro horas, e pelas tardes escrevia um artigo para algum jornal, e aqueles que conhecem a pressão de um artigo semanal, enquanto estão envolvidos com outro trabalho da imaginação, vão reconhecer a severa provação que Zola aguentou durante anos com muita persistência. Tinha pouco tempo para a reflexão ou para o estudo, e só conseguia inteirar-se das ideias que circulavam na época se elas passassem por ele. Lia seus contemporâneos, Flaubert, Goncourt, Daudet, e para obter um tablado de onde pudesse pregar sua doutrina, lia Balzac e Hugo; mas com relação ao coração da literatura francesa, com Montaigne, Saint-Simon e La Bruyère, é de se duvidar se seu conhecimento vai além do rudimentar. A influência de Manet, de Flaubert e de Goncourt o convenceu de que ele se interessa pelo mundo exterior, e nós consagramos A Taberna como uma obra-prima, pois quisemos nos unir em torno de um grande escritor. Abraçamos a causa de que, liberto de preocupações financeiras, ele iria ler,

${ }^{26}$ Compositor francês Alfred Bruneau (1857-1934). Amigo pessoal de Zola, os romances do autor serviram de base para a composição de oito óperas, dentre elas, La Rêve (1891), L'Attaque du moulin (1893), Messidor (1897), L'Ouragan (1901) e La faute de l'abbé Mouret (1907). Disponível em: <https://bit.ly/2MgwKmq>. Acesso em 17 jan. 2019.

27 "suspiros". 
refletir, viajar e se abster da produção constante, levando três ou quatro anos para compor e escrever um livro. Acreditamos que ele cultivaria a sofisticação do pensamento e a sofisticação da expressão literária. Mas Zola não era um artista por natureza. Em vez de os romances se tornarem mais e mais bonitos, eles tornaram-se maiores, mais frouxos e mais feios, e não tinham qualquer propósito, exceto conseguir dinheiro para a compra de santos vesgos com fundo de ouro.

Ai, as torres ridículas de Médan! Ai, a chegada de tradutores do Paraguai! Ai, o som dos trompetes diante do prefeito de Londres em homenagem ao A Terra [La Terre], A Derrocada, O Dinheiro [L'Argent] e O Doutor Pascal [Docteur Pascal]!

E três vezes, $\mathrm{Ai}$, pois não estamos agora sendo ameaçados por uma série de romances sobre Lourdes, Roma e Paris ${ }^{28}$ ? Ele vai reescrever nesses romances tudo o que já escreveu. Seus amigos vão se afastar; ele vai ficar sozinho; seus excelentes cigarros não vão nos atrair, e ao fumar alguns de péssima qualidade no café, vamos nos lamentar sobre a sua vida e o seu trabalho, e sobre o erro que cometemos; e quando o café fechar, ficaremos parados na beira da calçada, nos perguntando qual será o final. Um de nós dirá, provavelmente Huysmanns: "Em $O$ Ventre de Paris [Le Ventre de Paris], há um açougueiro de carne de porco que, após trabalhar dez horas por dia durante toda a sua vida, é encontrado morto, sentado em uma mesa son nez dans le boudin" 29 .

"E você acha", direi, "que ele vai cair de completa exaustão na sua mesa de trabalho son nez dans le boudin?"

Huysmanns não vai responder, ele se lembrará de que Zola é o grande amigo da sua vida. O pequeno grupo vai se separar, e caminhando até o meu pequeno apartamento na Rue de la Tour des Dames, lembrarei de Zola como o exemplo mais surpreendente da insanidade do bom-senso.

\section{ORIGINAL}

Manet had persuaded me to go to the bal de l'Assommoir dressed as a Parisian workman, for he enjoyed incongruities, and the blouse and the casquette, with my appearance and my accent, appealed to his imagination. "There is no Frenchman living in London who occupies the same position as you do in Paris", he said, and I pondered over his words as I followed him through tout Paris assembled at the Elysée Montmartre, for the ball given in honour of the play that was being performed at the Ambigu. "But I must introduce you to Zola. There he is", he said pointing to a thickly built, massive man in evening clothes for, as Manet said, a serious writer cannot be expected to put on fancy dress.

Zola bowed and passed on, chilling us a little; Manet would have liked to watch him struggling into a new acquaintanceship, and we walked on together conscious

${ }^{28}$ Alusão ao ciclo de romances intitulado Trois Villes [As Três Cidades], escrito no período de 1894 a 1898.

29 "com o nariz no chouriço". 
of our failure, myself thinking how pleasant it would have been to have gone with them into a corner, and talked art for half an hour, "and what a wonderful memory it would have been!" I thought, and begged Manet a few minutes later to come with me in search of Zola. But he was nowhere to be found.

"He must have gone home", I said, and Manet answered: "It doesn't matter. You'll find him at home at Mèdan any day you like to go there".

For one reason or another it was not till some months later that I summoned courage and took the train at the Gare St Lazare. There is no station at Mèdan, the nearest station is - (the curious are referred to the time table for I have forgotten the name of it) - and Mèdan is a village known only to peasants, about a mile and a half from the station. Some chance had led Zola there, and being in want of a country residence he had purchased a cottage from one of the peasants, which he had just finished building into a sort of castle; an ugly place it seemed to me, a great red brick wall with a small door in it through which I was taken into the house, and left waiting in the billiard room.

It was not Zola that came down to me, but Madame Zola. She had forgotten me, I had met her at Manet's studio, and it was not only after many tedious explanations that she somewhat reluctantly led me through the house, up many staircases of polished oak, narrow and steep. On the wall of the last little flight there were Japanese prints depicting furious fornications; a rather blatant announcement, I thought of naturalism - but they were forgotten quickly, for in a few seconds I should be in the master's presence. She opened a door and left me, and I found myself in a place as large as the studio of an Academician, lighted by a skylight and a huge window. For a moment or two I lost my way among the massive furniture, and it was not until I passed a lectern that I discovered the master on the sofa by a window correcting proofs.

He did not rise to meet me, but contented himself with untucking his fat leg and motioning with his hand to a seat. His manner was terribly aloof and cold, and my embarrassment increased, for suddenly I remembered I had heard that Zola was never long in doubt as to whether he was talking to a fool or a man of wit, and that at the end of a minute a fool was dismissed peremptorily. "And he has discovered me to be a fool though I haven't said a word". I glanced at the terrible master who lay on the sofa, his glasses on his nose, reading me, divining the common-place remarks that I was trying to conjure up. "If Homer and Shakespeare were suddenly introduced they would have to begin with remarks about the weather or the pleasure each had taken in the other's work" I said to myself, "and if this man would only give me as much rope as we would to Shakespeare or Homer I might think of something more interesting than the compliments that I am gabbling".

Zola was not then what he is now, a gracious, kindly man in the habit of receiving every one who chooses to call on him, and answering all sorts of question. He was then the iconoclast, the idol-breaker, a bear that cursed the universe, and bade all comer begone. All the same his writing exhaled a certain large-heartedness and 
sympathy, and I had always felt while reading his weekly article in The Voltaire that we were intended to understand each other. I had imagined that when I went to see him he would come forward, his hands extended in benevolent gesture, taking me at once into his confidence. This Buddha lying on the sofa, fixing his glasses from time to time on his short, strangely square-cut nose, was in such strange conflict with my dream that I could hardly believe that this could be my Zola in those terrible moments during which I tried to improvise compliments. Not one had produced the faintest impression, no more than water flowing over a block of granite, and feverishly I sought for a subject of conversation, something, no matter what, that might interest him.

The power that the circulating libraries exercised on literature occupied my mind a good deal at that time, and I hurried to the subject, seizing the first transitional phrase.

"The position of the novelist in England is that of a slave", I said, "for books are not bought in England, but hired".

"But if a man writes a book that interests the public, the public will find it".

"The public will find it in time no doubt", I answered, "but the man may starve in the interval".

"Yes, he may; and your difficult is no small one, a middle-man always between you and your public".

"Ah, he's beginning to see I'm not such a fool after all”, I said to myself, and as soon as I had explained the power that their monopoly gave to Mudie and Smith I deflected the conversation dexterously from the practical to the moral question, dropping some disparaging remarks about Puritanism as an artistic influence. Zola, who had been waking for some timeout of his slumber, was now wholly awake.

"What you say", the great man said, "is extremely interesting. I have written an article on the influence of Protestantism on art; it will appear in to-morrow's Figaro, and I make this statement, that Protestantism has never produced great art. Milton is the one Protestant writer. The Elizabethans, Shakespeare, and Jonson lived before Protestantism had taken hold of the national spirit, the genius of the nation, and so on".

The conversation then became friendly and pleasant. Zola asked me about George Eliot; which did I think was her best book? What French writer was she most like? Though I felt I was risking my newly-acquired reputation, I had to admit that I could think of no one to compare her with. The conversation paused a moment, and to my surprise and pleasure Zola began to tell me about the novel he was writing. We must have talked for three-quarters of an hour, and then, fearing to outstay my welcome, I bade the master good-bye. He took me downstairs, vivacious all the time, and asked me to come to see him again. Then I knew I had made a friend. "I have made a friend", I repeated to myself as the carriage rolled through the flat, green French country, my eyes noting the mystery of the long low horizon, the poplars pointing to the first stars. A train shrieked across the solitude. "I have made a 
friend", I repeated to myself as I listened to the distant rattle, and as the rattle died away in faint echoes, absorbed one by one in the dusky night of the long low plain I said: "Yes, he is the very man I had imagined from reading his articles. A clear, wellbalanced mind, a sympathetic nature, passionate in his convictions, loyal to his opinions. A little roughness at first; possibly what I mistook for roughness was mere shyness; besides it cannot be amusing to be told to your face that you are a great writer. I shouldn't like it myself'.

Years passed. I had written many books. A Mummer's Wife had been translated into French; it had been published in The Voltaire and the Vie Populaire. Charpentier was about to issue it in book form, and Zola had promised to write a preface. The Confessions of a Young Man was appearing in La Revue Independante, and the report had gone abroad that the next instalment would contain a scathing attack on La Terre. I wrote to Zola saying that this was not true, and proposing to spend Monday with him. "On Monday morning you will receive the new number of the Review, and we shall be able to discuss the matter at breakfast". I knew that the number contained - well, some frivolous remarks about naturalism; these I hoped to be able to explain away. But I did not feel quite at ease, so I called on my way for the faithful Alexis - bulky Alexis placid temperament would serve as a buffer when the discussion became strained. However happily it might end there could hardly fail to be moments when - I don't think I finished the sentence at the time; I will not seek to do so now.

Our walk lay by the river shimmering like watered silk between green banks full of the lush of June, and beyond them the green French country seemed to rejoice in the sunshine like a living creature. We sauntered, talking about our books, and I took exquisite pleasure in the poplars growing so tall and straight out of the plain, and the white clouds hanging between the trees. And when remembrances of Zola interrupted my reveries I told Alexis exactly what I had written, and the dear fellow assured me that Zola could not take offence at such light criticism.

"Yes, Alexis, but you always say what is agreeable to hear".

As before, Zola was lying on the sofa by the window and after a few words of greeting, he said:

"I'm afraid, my dear friend, that I shall not be able to write the preface. You have made it impossible for me to do so".

The phrases I had used when subjected to a close critical examination proved more difficult of explanation than I had anticipated. The discussion was painful, and the breakfast bell was a welcome relief. "It's over at last", I said to myself; but to my horror instead of answering my thought the master said:

"We are going down to breakfast now, but after breakfast we will go into the matter thoroughly; I will read the passages aloud to you".

"Good Heavens!" I thought, "I wish I hadn't come".

After breakfast Zola, Alexis, and myself walked in the garden talking of indifferent things for an hour or more. Then Zola said: 
"We will now go upstairs". He led the way, and I followed, feeling very much as I used to feel at school when ordered a flogging. The master lay on the sofa; I took a small chair; he said: "You'll be more comfortable in a larger one".

The passages were already marked, and they were read to me in a low and deliberate voice. I listened, thinking what was the best defence to set up; Zola commented on every fresh sarcasm.

"How can I write your preface after that? I want to, you know, but I ask you how can I? Listen!"

"Don't you see, my dear friend, that that book is not my real opinion about life and things, but rather an attempt to reduce to words the fugitive imaginings of my mind, its intimate workings, its shifting colours? Has it never come to you to think differently about things? To find your mind in a ferment of contradiction?"

"No", he said, "I do not change my opinions easily. There is Alexis" (he was indeed there, round as a barrel with the inevitable cigar between his teeth); "I have known Alexis these five and twenty years, and I think of him today exactly as I always thought of him. With me an opinion is like a heavy piece of furniture; it is moved with difficulty".

"But", I said, "the passages you have just read are from a chapter entitled 'La Synthèse de la Nouvelle Athenes'...and must be taken as an expression of the opinions of the various rates who assemble there".

"I will admit that as a legitimate defence, but you see the opinions expressed in the café coincide exactly with those you express yourself in an earlier part of the book".

I had to fall back on the original defence, that a man changes, contradictory thinking should not be taken for the opinions which he holds by and abides by.

"How often do we hear Christians make jokes against Christianity?" I thought the argument specious, but Zola did not notice it. He continued reading:

"After you have written about Goncourt", he said, "you never can go to this house again".

"I don't want to; he isn't a friend".

"The disciples, the childish vanity, the bric-à-brac, even the accusation of making copy out of his brother's corpse, tout est lá, rien ne manque. What you say of me is nothing compared with what you say of Goncourt". I hastened to concur in this opinion, but Zola was not to be wheedled. "No, my dear friend," he said gravely and sadly, "you don't call your book Memoirs d'un Jeune Anglais you say Confessions d'un Jeune Anglais, and when we use the word Confessions we mean that last we are going to tell the truth. I have gone through theses pages calling attentions to the expressions used, not because I am angry, but because I want to convice you that you have made it impossible for me to write the preface to your A Mummer's Wife. What you think of me does not affect me, no, I won't say that; we are old friends. What you say about me does affect me, I mean that nothing that you can say can affect my position...You admit in your book that you owe your first 
inspiration to me. I am proud that this is so, and thank you for saying it. I am sorry you have changed your opinions; after all it is the eternal law - children devour their fathers. I make no complaint. Nature has willed it so".

He spoke these words sadly as he walked across the room. The twilight was gathering, the great furniture loomed up like shadows. There were tears in my eyes. Never did I feel so distinct a sensation of my inferiority; the man was great in his simplicity. "The man is greater than his books", I said to myself, "and that is a great deal, for he was written some very fine books".

I have told the story of these two meetings with some levity, but I was deeply moved at the time, and I am troubled even now, for is it always right to wear one's heart on one's sleeve, and to publish one's opinions as they come up in one's mind? Or is it better to look upon one's opinions as heavy pieces of furniture that are moved with difficulty. Alexis had devoted months to the correction of the translation that Charpentier was about to issue, and looked to Zola's preface to recoup himself for the labour he had spent upon the book, and a few casual words of mine had wrecked these hopes. He did not reproach me with having cost him some monetary losses; he merely said, "C'est Charpentier que va boire un bouillon. Mille francs de corrections". The grey-green country stretched out before us, flat and dim - a dark mass of trees in front of us, a poplar striking out of the long plain. Alexis lectured me as we walked through the solitary country, but I did not listen. All the while I thought of Zola's last words as he bade me good-bye. "I hope you understand that out personal relations are the same as they always were, only you have made it impossible for me to write the preface".

At this time Zola was a fat man; soon after he became a thin one. By abstaining from drink at his meals he reduced his weight thirty-six French pounds in two months. He seems to have accepted Balzac's maxim, that the elegance of life exists mainly in the waist. As his waist narrowed his manner of life became more expansive. No longer is he the recluse of Mèdan; he had added a tower to his country house - with what intention I never fully understood - and he lives in a spacious mansion in the Rue de Bruxelles, which he has furnished with oak carvings, tapestries, portraits of archbishops and wrought-iron railings. A plaster cast of the Venus de Milo stands on the balustrade that encircles the staircase. The house seems to reveal a large coarse mind, a sort of coarsely woven net through whose meshes all live things escape, and that brings to shore only a quantity of débris. "From the Rue de Lafayette", I said. Why should he consider it incumbent upon him to collect these things? Great artists need not be learned in bric-à-brac. Manet lived all his life amid red plush furniture; and I am not sure that I should have spoken of Zola's furniture (has it not been described by reporters and reproduced in photography in every illustrated periodical?) if it had not been that with the acquisition of a waist and much general bric-à-brac a definite mental change has come upon Zola. I once heard him say he was going to give a ball. I don't think he ever carried the project into execution. However this may be, his house has, for the last three years, been open to 
visitors, and he has answered the ten thousand heterogeneous questions that the eleven hundred and fifty-seven interviewers have put to him with unfailing urbanity, and I am bound to admit, with extraordinary common-sense.

His mind is not as intense or penetrating as Tourguèneff's, but it looks with admirable lucidity over a wide surface, and he can answer the most foolish question reasonably. An elderly lady's applecart has been upset in the Place Cliche, and a reporter calls on Zola for his opinion. He says that he has no precise information on the subject of apples, but he believes that apple-growing is a very large industry in the north of France. If the apple sellers of Montmartre are prevented from exposing their wares for sale, the liberty of the individual is called into question, and a very large and important industry is possibly affected. At the same time the streets cannot - and so on. But even these platitudes he will relieve with some touch of rare common-sense. This touch I have left out, it is the incommunicable secret of his genius. But if any reader of this article should desire to hear Zola talk, I will recommend him to a book called Enquête Litteraire by Jules Huret. Huret's interview with Zola is an astonishing piece of literature. In this interview we perceive, as we should in a long intercourse with Zola himself, that his genius is but the triumph and apotheosis of common-sense. For his genius is wingless, it never rises towards the stars; it maintains itself at what I may term the level of superior mediocrity, and it is with him always, on small as on great occasions. Take his answer to an interviewer who called on him at the Savoy Hotel. Zola had arrived late the night before, and had only just got out of the bed. The question was: "What are your impressions of London?" The answer was: "My first impression of London was an excellent appetite. The train was late, and we didn't dine until nine o'clock, but we dined excellently well". Is it possible to answer a foolish question more sensibly?

I said just now that Zola's vision of life was not so intense, penetrating, or subtle as Tourguèneff's. It is radically different. Zola's mind is patriarchal; he is and oldworld hero, a patriarch belated in the nineteenth century. Not Abraham himself encamped amid his flocks, herds, and a numerous servitude saw or thought more simply than he does. There are hackney carriages, washerwomen, and châssepots in the Rougon Macquart series, but these are merely adventitious attractions which affect in no way the general character of the work. Hugo is said to be the last of the old-world poets; but the real difference between Zola and Hugo is that one can, and the other cannot, write verse. Take from Hugo his genius of versification and you would get the novelist. He would have produced a set of novels very similar to the Rougon Macquart series. It would have been in twenty volumes, possibly in more, and would have sold as largely. Robbed of his versification, Hugo would have accepted the hackney carriages and the washerwomen. He could not have done otherwise, and both men saw life from the outside and their tendency was to exaggerate the outside. All the same the hypothetical work would have differed from the Rougon Macquart. Hugo was more naturally and artist than Zola. His imagination was rarer, but it was not more powerful nor more fecund. Zola's imagination is one of 
the most extraordinary that ever found expression in literature. Think, you who have read the twenty volumes, of the hundreds of places he has shown you and familiarised you with, even as you are familiar with the room you live in. Can you not see incestuous Renée dreaming in her yellow boudoir, or feverishly flung on the skins under the malign shade of tropical plants in the great conservatory? Are not the market-places in your mind - the roofs for ever silhouetting against the pale sky? The smell of the fish and the hundred colours of the fish; the vaults where children roll amid the feathers? And the scene where they chop the pigeons' heads, disputing how many so-and-so can bleed in an hour? And how intimately conscious we are of the great garden of the Paradou and the adorable death of Albine, who dies asphyxiated by the flowers with which she has filled the room; the enumeration of the flowers, the evocation of an orchestra of scents, for every scent recalls the sound of an instrument, and the last phrase - "Albine dies in a supreme hiccup of flowers" - how wonderful!

And the modes of life, the trees, the various ways in which the human animal gains his livelihood! Do we not all remember the gold chain-makers in L'Assommoir and the vestment makers in Le Rêve? In Au Bonheur des Dames the work of every employé is explained; the phenomena of each passing hour is revealed to us. Germinal is full of every detail of miner life, the ropes, the pulleys, the furnaces, the trucks, the horses. But I must stay my pen or this article will degenerate into a mere catalogue.

But has Zola furnished these extraordinary evocations of the external of human life with human souls? Has he created characters that will not suffer by comparison with Balzac's? Zola's evocation of souls is slight, nearly always fragmentary and shadowy. A soul haunts in Gervaise, and Coupeau, too, has a soul, and through the numberless pages a few shades flit vaguely recognisable as human souls. That is all. In the line of souls Gervaise is his greates achievement, and that is why I place L'Assommoir above all his other books. There are other reasons. When he wrote L'Assommoir Zola was more than he ever was before, and certainly more than he ever was since, a pupil of Flaubert. The book is written entirely in Flaubert's manner, the short sentences relieved by the pictorial epithet. The old masters thought that originality was found in individual feeling and seeing rather than in mannerism, and as I share their opinion I think that it is regrettable that Zola did not continue to write in the style in which he produced his finest book. But the style became too laborious for him just as pre-Raphaelism became too laborious for Sir John Millais, and after L'Assommoir his style became looser, and with every fresh book he seems more and more inclined to abandon himself to the ease of redundant expression. There are fine pages even in his worst books, but so far as my personal taste and interest are engaged in his work I would choose to have revised editions of his early works rather than the new novels he contemplates writing - Lourdes, Paris, Rome, etc.

Revised editions of Zola's works! How easily one drops into talking nonsense. His method of novel-writing does not admit revision. As well might we ask the 
editor of a daily paper for concentration of expression in leading articles, dramatic notes, and reports of boat races. During the last ten or a dozen years a striking resemblance has grown up between Zola novel and the popular newspaper. The novel and the newspaper seem to me to stand on the same footing; the intention of both is the same, and the means employed are the same. It is true that Zola's reports on the Franco-German war are better done than the reports of the war correspondent of the Daily Telegraph. It is also true that the scenery at the "Lyceum" is better painted than the scenery at the "Surrey", but that is hardly a reason for confusing a set taken from Much Ado about Nothing with the pictures of Turner, Constable, and Wilson, and we find a like difference between the battle pieces in War and Peace and those in La Débâcle - a difference not of degree but a difference of kind. Zola's novel is practically the daily paper. He has discovered a formula that suits the average man as well as the Daily Telegraph or the Petit Journal, and he chooses his subjects, not in obedience to an artistic instinct, but in accordance with public taste. Three hundred thousand pilgrims go to Lourdes yearly. Every pilgrim is a certain reader, and the afflicted in all countries are interested in the question. Between belief and unbelief he will steer a middle course just as he steered a middle course between France and Prussia. I heard him boast, without ever perceiving the enormous artistic significance of what he was saying, that he had written a French novel on the war without giving Prussia cause for offence. I take it that the sublime impartiality of the true artist is very different from the mock impartiality of the journalist who wants to get up a controversy. The true artist sees life as God sees it, without prejudice; life is for him - I think the phrase is Flaubert's - une hallucination à transporter.

Zola told me that he had gone into calculation, and allowing for a fortnight's holidays at Christmas, Lourdes would take him seven months to write. Five hundred pages in seven months! Tolstoy took six years to write Anna Karenina, ten to write War and Peace; Flaubert took seventeen years to write La Tentation de St Antoine, eight or nine to write L'Education Sentimental; and seven or eight translators are already at work on Lourdes; it will appear in the New York Herald, and Mr. Bennet has paid a thousand pounds for the serial rights. But adequate information regarding the various forms and languages in which this book will appear would be the subject of an article on bibliography. Suffice it to say here that it will bring Zola something like four thousand pounds before it reaches Charpentier in book form; it will then be read by everybody except men of letters - but their number is so small that the abstention will not materially affect the sale. If the book does not sell three hundred thousand copies it is a failure, and if the book on the Russian Alliance which will follow does not sell half a million it will be a failure. Did any great writer ever see literature from this point of view before?

The idea of conquest seems inherent in Zola. Five and twenty years ago he wrote a book called Le Conquête de Plassans. The idea of conquest cropped up again in L'Oeuvre, and this time it was Paris that was conquered. And now it seems that Zola 
meditates the conquest of the world. He came to England at the head of an army of journalists; rockets were let off at the Crystal Palace, and trumpets were blown in his honour at the Mansion House. He will probably proceed on a similar mission to St Petersburg (it has already been spoken of); he may even visit America. Why not? There are sixty millions in the United States, who through the medium of translation, may read the Rougon Macquart! The newspapers reported that Madame Zola, astonished at the length of our London suburbs, said: "This is a town that would suit you, Emile". Every house represented to her a possible sale of a novel, Charpentier edition, three francs fifty. If Zola were told that a concierge had not heard his name he would feel discouraged. An enquiry would be set on foot, and if a concierge's guild could be discovered he would arrange to address a meeting. He looks upon all me who do not read his novels as lost. Lost to what? Ah, that I cannot say; not to art, for the quality of his writing does not seem to concern him any more than the quality of the things he buys. The carved woodwork and the iron railings may not be finely wrought, but the photograph all right, and every interviewer is received and every sightseer - Chinese, Peruvians, Esquimaux - all and sundry are granted audience, and the afternoon passes in talking of how books may be best put on the market.

Some translation of his works must appear in every dialect, and to discover one not yet reduced to written characters, and to arrange that the first work printed in it should be a translation of the Rougon Macquart series would be fame indeed. M. Bruneau comes in with the score of the music he has just written for one of the novels, and the gentleman from Paraguay jumps up and proposes to do the opera into the language spoken in his country; the Thibetian might to the same. Bruneau and Zola put their heads together. Hurrah! another outlet has been discovered, and the terms of the contract are discussed. Only the other day in an article on the lyrical drama, after coupling Wagner and Bruneau together, Zola explained that he would create a lyrical drama with human characters; and when he has done this "the colossal Wagner will grow pale on the high pedestal of his symbols". Zola believes that young French composers have not written great music because their libretti are not sufficiently human. In a word, he imagines himself writing various libretti to which the young French composers will add a little music as cream is added to méringues. Well, if a man will talk on all subjects the time will come when he will talk nonsense. I am afraid that time has come for Zola!

The desire of gold for its own sake is comprehensible in a way; but Zola has no love of money, he has squandered all he made on vulgar decoration and absurd architecture. The pleasures of life bore him exceedingly, so he says; but I am afraid that he has not acquainted himself with them. Of the pleasures of Art he is equally ignorant. His youth was beset with difficulties sufficient, be it admitted to his credit, to conquer all but the most resolute. He wrote for four hours every morning at a novel, and every afternoon he wrote an article for a newspaper, and those who have felt the pressure of a weekly article, while engaged on a work of the imagination, 
will appreciate the severity of the ordeal Zola bore for many years unflinchingly. He had little time for reflection or study, and was only able to catch the few ideas abroad in his day as they passed him. He read his contemporaries, Flaubert, Goncourt, Daudet, and to obtain a platform whence he might preach his doctrine he read Balzac and Hugo; but with the heart of French literature, with Montaigne, St Simon, and La Bruyère it may be doubted if his knowledge is more than rudimentary. The influence of Manet and Flaubert and Goncourt persuaded him that he was interested in the external world, and we hailed L'Assommoir as a masterpiece, for he wished to group ourselves round some great writer. We hugged the belief that, set free from pecuniary anxieties, he would read, think, travel, and refrain from constant production, giving three or four years to the composition and the writing of each book. We believed that he would cultivate refinement of thought, and refinement of literary expression. But Zola was not naturally an artist. Instead of the books becoming more and more beautiful, they have become larger, looser, and uglier, and they serve no purpose whatsoever, except to find money for the purchase of cock-eyed saints on gold backgrounds.

Alas! the ridiculous towers of Mèdan! Alas! the arrival of translators from Paraguay! Alas! the blowing of trumpets before the Lord Mayor of London in honour of La Terre, La Débâcle, L'Argent, and Docteur Pascal!

And, three times, alas, for are we not now menaced by a novel on Lourdes, on Rome, and on Paris? In these novels he will re-write everything that he has written before. His friends will drop away from him; he will be left alone; his excellent cigars will fail to attract us, and smoking bad ones in the café we shall regret his life and his works, and the mistake we made; and when the café we shall stand on the edge of the pavement wondering what the end will be. One of us will say, it will probably be Huysmanns: "In Le Ventre de Paris there is a pork butcher who, after having worked ten hours a day all his life is found dead sitting before a table son nez dans le boudin".

"And you think", I shall say, "that we will just drop from sheer exhaustion over his writing table son nez dans le boudin?"

Huysmanns will not answer, he will remember that Zola is the friend of his life. The little group will separate, and wending my way to my little flat in the Rue de la Tour des Dames, I shall think of Zola as a striking instance of the insanity of common-sense. 
FRAZIER, Adrian. "Preface". In: MOORE, George. A Mummer's Wife. Brighton: Victorian Secrets, 2011.

FURST, Lilian. George Moore, Zola, and the Question of Influence. Canadian Review of Comparative Literature, v.1, n.2, p.138-155, 1974.

MOORE, George. Confessions of a Young Man (1888). Gloucester: Dodo Press, 2007.

.My Impressions of Zola (1894). In: MOORE, George. Impressions and Opinions. Londres: T. Werner Laurie, LTD, 1913.

SHOWALTER, Elaine. Anarquia sexual: sexo e cultura no fin de siècle. Trad. Waldéa Barcellos. Rio de Janeiro: Rocco, 1993.

ZOLA, Émile. “O Romance Experimental”. In: O Romance Experimental e o Naturalismo no Teatro. Trad. e Notas de Italo Caroni e Célia Berrettini. São Paulo: Perspectiva, 1979. 